\title{
Role of fluttering dislocations in the thermal interface resistance between a silicon crystal and plastic solid ${ }^{4} \mathrm{He}$
}

\author{
Jay Amrit, ${ }^{1, *}$ Aymeric Ramiere,,${ }^{1, \dagger}$ and Sebastian Volz ${ }^{2}$ \\ ${ }^{1}$ Laboratoire d'Informatique pour la Mécanique et les Sciences de l'Ingénieur, CNRS, Univ. Paris-Sud, \\ Université Paris-Saclay, Rue John von Neumann, 91405 Orsay, France \\ ${ }^{2}$ Laboratory for Integrated Micro Mechatronic Systems, CNRS-Institute of Industrial Science, University of Tokyo, Tokyo 153-8505, Japan
}

(Received 6 August 2017; revised manuscript received 15 October 2017; published 22 January 2018)

\begin{abstract}
A quantum solid (solid ${ }^{4} \mathrm{He}$ ) in contact with a classical solid defines a new class of interfaces. In addition to its quantum nature, solid ${ }^{4} \mathrm{He}$ is indeed a very plastic medium. We examine the thermal interface resistance upon solidification of superfluid ${ }^{4} \mathrm{He}$ in contact with a silicon crystal surface (111) and show that dislocations play a crucial role in the thermal interface transport. The growth of solid ${ }^{4} \mathrm{He}$ and the measurements are conducted at the minimum of the melting curve of helium $(0.778 \mathrm{~K}$ and $\sim 25 \mathrm{bar})$. The results display a first-order transition in the Kapitza resistance from a value of $R_{K, L}=(80 \pm 8) \mathrm{cm}^{2} \mathrm{~K} / \mathrm{W}$ at a pressure of 24.5 bar to a value of $R_{K, S}=(41.7 \pm 8) \mathrm{cm}^{2} \mathrm{~K} / \mathrm{W}$ after the formation of solid helium at $\sim 25.2$ bar. The drop in $R_{K, S}$ is only of a factor of $\sim 2$, although transverse phonon modes in solid ${ }^{4} \mathrm{He}$ now participate in heat transmission at the interface. We provide an explanation for the measured $R_{K, S}$ by considering the interaction of thermal phonons with vibrating dislocations in solid ${ }^{4} \mathrm{He}$. We demonstrate that this mechanism, also called fluttering, induces a thermal resistance $R_{F l} \propto N_{d} T^{-6}$, where $T$ is the temperature and $N_{d}$ is the density of dislocations. We estimate that for dislocation densities on the order of $\sim 10^{7} \mathrm{~cm}^{-2}, R_{F l}$ predominates over the boundary resistance $R_{K, S}$. These fundamental findings shed light on the role of dislocations and provide a quantitative explanation for previous experiments which showed no measurable change in the Kapitza resistance between $\mathrm{Cu}$ and superfluid ${ }^{4} \mathrm{He}$ upon solidification of the latter. This demonstrates the possibility of using dislocations as an additional means to tailor thermal resistances at interfaces, formed especially with a plastic material.
\end{abstract}

DOI: 10.1103/PhysRevB.97.014308

\section{INTRODUCTION}

Heat flow across a solid/solid ${ }^{4} \mathrm{He}$ interface is original in that solid ${ }^{4} \mathrm{He}$ is a quantum solid. The atoms in solid ${ }^{4} \mathrm{He}$ have a large zero-point motion, approaching almost $25 \%$ of the lattice spacing. Also, recent studies [1-5] on the quest for supersolidity of ${ }^{4} \mathrm{He}$ in the group of Balibar and co-workers have revealed that its elastic properties are strongly impacted by the gliding motion of dislocations along the basal plane [6] of the hcp solid ${ }^{4} \mathrm{He}$ crystal, which is in contact with a classical solid surface. This defines a new class of interfaces thanks also to the giant plasticity of solid ${ }^{4} \mathrm{He}$. There is therefore a fresh interest in studying these interfaces and in observing how their quantum features may influence thermal interface properties, and in particular, the Kapitza resistance $R_{K}$.

An earlier interest in studying solid/solid ${ }^{4} \mathrm{He}$ interfaces was triggered from a quest to understand the enigmatic Kapitza resistance at a solid/superfluid ${ }^{4} \mathrm{He}$ interface. Indeed, liquid helium at low temperatures is one of the rare pure materials whose phase state can be monitored by mechanical pressurization at a constant given temperature. The phase change from superfluid

\footnotetext{
*Corresponding author: jay.amrit@limsi.fr

${ }^{\dagger}$ Present address: Department of Physics, South University of Science and Technology of China, Shenzhen 518055, People's Republic of China.
}

to solid ${ }^{4} \mathrm{He}$ leads to a discontinuous increase in its density by $\sim 20 \%$. Transverse modes are now present in solid ${ }^{4} \mathrm{He}$ and they play a direct role in phonon transmission, thereby increasing the amount of thermal energy carried across the interface. Rotons, which are elementary excitations present in superfluid helium, disappear abruptly with phase change. A literature survey reveals a scarcity in the studies of $R_{K}$ upon solidification of helium. To our knowledge the only experiments conducted between a classical solid and solid ${ }^{4} \mathrm{He}$ are those of Folinsbee and Anderson [7] and Mezhov-Deglin [8]. Both experiments were conducted for copper/solid ${ }^{4} \mathrm{He}$ interfaces. And, both experiments led to another enigmatic result which is the absence of a change in $R_{K}$ when ${ }^{4} \mathrm{He}$ is either superfluid or in the solid phase.

Significant progress in the understanding of the Kapitza resistance $R_{K}$ between a silicon crystal (dielectric solid) and pressurized superfluid ${ }^{4} \mathrm{He}$ has been made by considering a new mechanism of resonant scattering of phonons in collision with the surface [9], as first predicted by Adamenko and Fuks [10]. We have demonstrated that resonant scattering occurs when $\sigma \approx 0.33 \lambda_{\text {HeII }}$ (see Fig. 3 in Ref. [9]) where $\sigma$ is the rms surface roughness height and $\lambda_{\text {HeII }}$ is the dominant phonon wavelength in the superfluid.

In this paper we examine the thermal interface resistance between Si crystal surface and helium solid at the minimum of the melting curve of ${ }^{4} \mathrm{He}$. We discover a distinct firstorder transition in the thermal interface resistance with the 


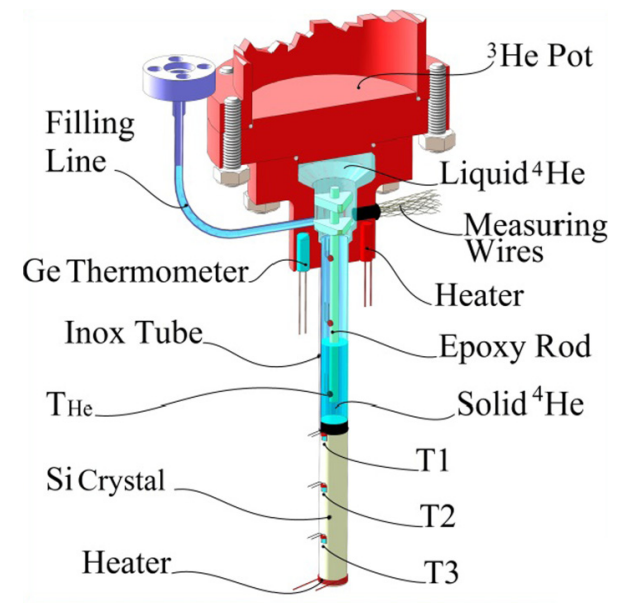

FIG. 1. Cell configuration for measurement of the Kapitza resistance at $\mathrm{Si} /$ solid ${ }^{4} \mathrm{He}$ interface. Solid ${ }^{4} \mathrm{He}$ is grown at the minimum of the melting curve from the superfluid phase.

solidification of helium, contrary to the results of Folinsbee and Anderson, and Mezhov-Deglin. We analyze these measurements by combining the two recent path-breaking findings related to the resonant scattering mechanism by surface roughnesses [8] and to the interaction of thermal phonons with vibrating dislocations in plastic solid ${ }^{4} \mathrm{He}[1-4]$. We show that dislocation densities on the order of $10^{7} \mathrm{~cm}^{-2}$ suffice to create thermal resistances in solid ${ }^{4} \mathrm{He}$ which predominate over $R_{K}$. Our analysis gives a coherent explanation for all experimental data, including those of Folinsbee and Anderson, and Mezhov-Deglin.

This paper is organized as follows: Section II describes the experimental setup, crystal-growth technique, and measurements. Section III analyzes the experimental data and shows that the nature of the scattering is predominately diffuse or resonant as in the case of the $\mathrm{Si} /$ superfluid ${ }^{4} \mathrm{He}$ interface. A major part of this section quantifies the role of dislocations (core and screw) in solid ${ }^{4} \mathrm{He}$ and the contribution of phonon interaction with mobile dislocations is clearly established as a function of the dislocation densities. Section IV concludes the paper.

\section{EXPERIMENTAL}

\section{A. Cell and experimental configuration}

The experimental cell is shown in Fig. 1 and it has been used in previous experiments [9]. The polished extremity of the single-crystal $\mathrm{Si}$ rod is in contact with helium present in a thin-walled tube which is filled using a stainless-steel capillary having an inner diameter of $0.2 \mathrm{~mm}$. This filling line is thermally anchored below the $1 \mathrm{~K}$ pot of a helium- 3 refrigerator. The $1 \mathrm{~K}$ pot is run at a temperature of $\sim 1.4 \mathrm{~K}$. The top part of the cell is made of copper and is tightly attached to the cold source of the refrigerator. The cell is equipped with six $\mathrm{RuO}_{2}$ thermometers, of which three are equally spaced on the Si crystal and the other three are in the tube. A heat flux generated with the use of manganin wire at the bottom extremity of the silicon crystal propagates along the $c$ axis of

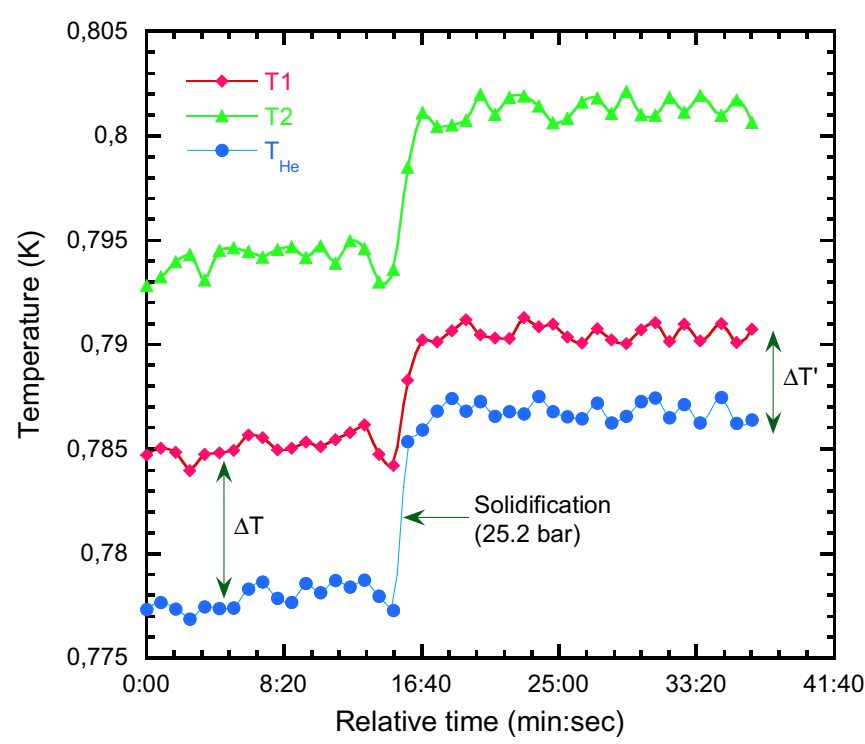

FIG. 2. Temperature evolution during growth of ${ }^{4} \mathrm{He}$ crystal under a constant heat flux. Solidification is accompanied by a change in temperature at the interface as shown by the $\mathrm{RuO}_{2}$ thermometers $T_{\mathrm{He}}$ and $T_{1}$. The temperature jump at the interface changes from $\Delta T$ to $\Delta T^{\prime}$ under a constant heat flux.

the crystal. Further details of the cell and the heat loss analysis are given in Ref. [11].

\section{B. Crystal growth and measurements}

The melting curve of helium-4 has a dip of approximately 8 mbar at a temperature of $0.775 \pm 0.012 \mathrm{~K}$ and a pressure of $24.985 \pm 0.005 \mathrm{~atm}$ [12]. For temperatures above the minimum point, the pressure in the cell is monitored directly by condensing helium through the gas-handling system. We used high-purity helium gas (Alphagaz ${ }^{\mathrm{TM}} 2$ supplied by Air Liquide, global purity $99.9999 \%$ ) in the experiment. The gas is sent through a nitrogen trap to remove residual impurities before injection into the cell. To ensure that helium solid forms in the cell and on the Si surface, the superfluid ${ }^{4} \mathrm{He}$ in the cell is first pressurized to $\sim 22$ bar and then regulated to the minimum temperature. With the temperature controlled to within $\sim 1 \mathrm{mK}$ of the minimum temperature, the pressure in the cell is discontinuously increased at a rate not exceeding $\sim 0.2$ bar per minute with the aid of a needle valve. The pressure is read on a Bourdon manometer. All thermometers were recorded continuously as liquid helium was solidified. The estimated crystal growth rate did not exceed $\sim 0.48 \mathrm{~mm} / \mathrm{min}$. This filling-rate condition was respected so as to obtain a good-quality helium crystal. Generally, solid ${ }^{4} \mathrm{He}$ grown at the minimum temperature of the melting curve by slow pressurization of the cell produces a high-quality single crystal [13]. At the minimum point there is no latent heat release, eliminating thermal stresses in solid ${ }^{4} \mathrm{He}$ as it is grown.

\section{RESULTS AND DISCUSSION}

\section{A. Transition in $\boldsymbol{R}_{K}$ with phase change from superfluid to solid}

Figure 2 shows the trace of helium temperature given by $T_{\mathrm{He}}$ which is situated at a distance $\ell_{\mathrm{He}} \approx 2 \mathrm{~mm}$ from the interface. 


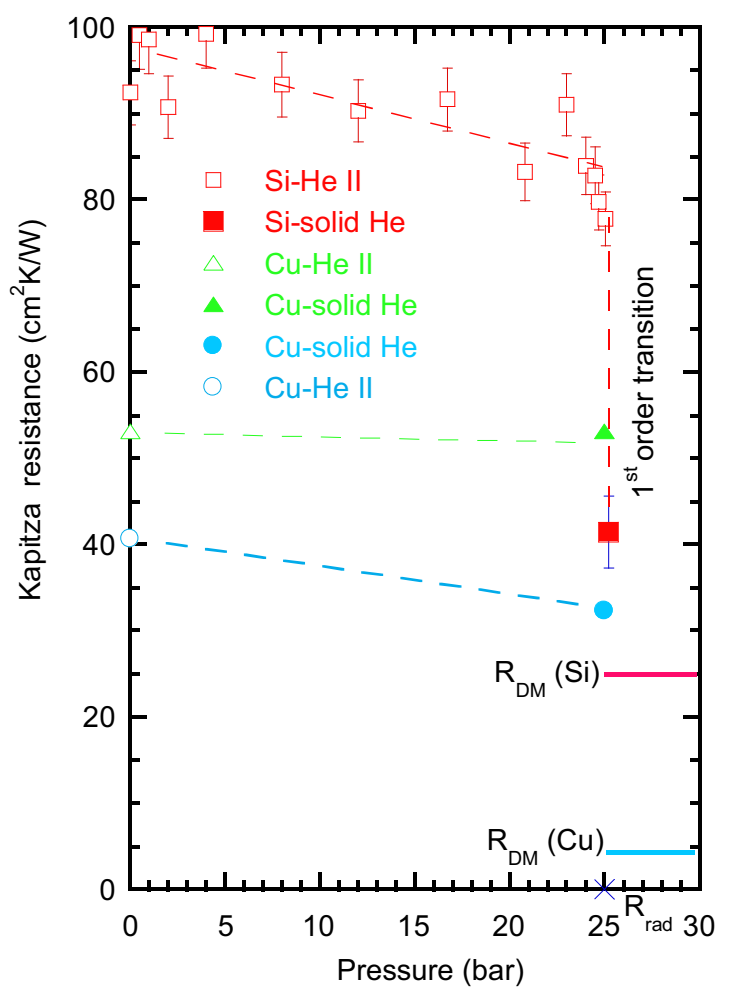

FIG. 3. Measurements of $R_{K}$ at the minimum of melting curve. Open symbols: $R_{K}$ between a solid and superfluid. Full symbols: $R_{K}$ between a solid and solid ${ }^{4} \mathrm{He}$. For the $\mathrm{Si} / \mathrm{He}$ interface: $R_{K}$ gradually decreases as the pressure varies from SVP to 25.2 bar (open red squares). The first-order transition is observed upon solidification (full square) as highlighted by the dashed red line. For the $\mathrm{Cu} / \mathrm{He}$ interface: the triangles are measurements of Mezhov-Deglin (Ref. [8]) and the circles are taken from works of Folinsbee and Anderson (Ref. [7]). Here the dashed lines are visual guides to indicate the absence of a transition in $R_{K}$ upon solidification. The continuous blue and red lines are the diffuse mismatch model predictions of $R_{K}$, respectively, for $\mathrm{Cu}$ and $\mathrm{Si}$ in contact with solid ${ }^{4} \mathrm{He}$. The $(x)$ indicates the radiation limit model prediction.

Also shown in this figure is the temperature evolution along the silicon crystal, depicted by $T_{1}$ and $T_{2}$, which are situated, respectively, at 2 and $12 \mathrm{~mm}$ from the interface. When superfluid is present in the cell, its temperature is that of the regulated temperature of the copper part of the cell, which is attached to the cold source. $T_{\mathrm{He}}$ remains constant at all times even when the heat flux directed from the crystal across the interface is modified. Throughout this experiment, a small heat flux less than $30 \mu \mathrm{W}$ is applied across the interface and it is maintained constant. The solidification process is clearly observed as it provokes a temperature shift on all thermometers as shown in Fig. 2. The change in the temperature difference before and after solidification from $\Delta T=\left(T_{\mathrm{He}}-T_{1}\right)$ to $\Delta T^{\prime}$ is directly proportional to the change in $R_{K}$ with the crystallization of ${ }^{4} \mathrm{He}$. The Kapitza resistance at the $\mathrm{Si} /$ solid ${ }^{4} \mathrm{He}$ interface is given by $R_{K, S}=R_{K, L} \times\left(\Delta T^{\prime} / \Delta T\right)$.

Figure 3 shows the measurements of the Kapitza resistance as a function of the pressure in the superfluid as the latter approaches solidification. Details of typical measurements before solidification are given in Ref. [9]. The Kapitza resis- tance undergoes a clear jump upon solidification of the superfluid. Just before the transition, $R_{K, L}=(80 \pm 8) \mathrm{cm}^{2} \mathrm{~K} / \mathrm{W}$. After solidification, the Kapitza resistance reaches a value of $R_{K, S}=(41.7 \pm 8) \mathrm{cm}^{2} \mathrm{~K} / \mathrm{W}$. This change corresponds to an instant $\sim 48 \%$ drop in the Kapitza resistance.

The thermal boundary resistance clearly undergoes a firstorder transition. The order parameter here is the helium density and the order variable is the thermal boundary resistance $R_{K}$ or the average transmission coefficient. The sharp increase in the transmission, as shown later, is due to the fact that there are more modes present in solid ${ }^{4} \mathrm{He}$ than in the superfluid. Indeed, the presence of transverse branches in solid helium allows for a better coupling to silicon.

\section{B. Predictions of models for interface resistance \\ 1. A modified acoustic mismatch (AM) model for strongly mismatched solids}

To analyze our results we developed a simple modified version of the AM model for solids having very large discrepancies in their acoustic impedances. In our model (see Supplemental Material [14]) the thermal interface resistance is given by

$$
R_{\mathrm{AM}}=\frac{1}{4 a T^{3}\left(1-\cos \theta_{c}\right)}\left[\frac{\alpha_{L}}{c_{\mathrm{He}, L}^{2}}+\frac{2 \alpha_{T}}{c_{\mathrm{He}, T}^{2}}\right]^{-1},
$$

where the transmission coefficients $\alpha_{L}=\left(4 z_{1, L} z_{2, L}\right)$ $\left(z_{1, L}+z_{2, L}\right)^{-2}$ and $\alpha_{T}=\left(4 z_{1, T} z_{2, T}\right)\left(z_{1, T}+z_{2, T}\right)^{-2}$ with the acoustic impedances $z_{i, j}=\rho_{i} c_{i, j}, i=(1,2)$ refer to medium 1 and 2; and $j=(L, T)$ refers to the longitudinal and transverse phonon branches, respectively. The key feature is the use of a unique critical angle $\theta_{c}$ which is the average of the critical cone angles $\theta_{c, L}$ and $\theta_{c, T}$, associated, respectively, with the $L$ - and $T$-phonon branches in the less-dense medium (solid He in our case). Using appropriate values for $\rho_{i}$ and $c_{i, j}$ (see Supplemental Material), our modified AM model prediction of the Kapitza resistance between solid ${ }^{4} \mathrm{He}$ and $\mathrm{Si}$ therefore behaves as

$$
R_{\mathrm{AM}}=\frac{0.89}{T^{3}\left(1-\cos \theta_{c}\right)} \mathrm{cm}^{2} \mathrm{~K} \cdot \mathrm{W}^{-1} .
$$

Equation (4) infers a rapid growth in the thermal boundary resistance as $\theta_{c}$ tends to 0 for materials having very different sound velocities.

In solid ${ }^{4} \mathrm{He}$ we found $\theta_{c} \approx 3^{\circ}$, which yields $R_{\mathrm{AM}}=$ $1371 \mathrm{~cm}^{2} \mathrm{~K} / \mathrm{W}$ at $T=0.778 \mathrm{~K}$. This predication is approximately 30 times our measured value of $R_{K, S}=(41.7 \pm$ 8) $\mathrm{cm}^{2} \mathrm{~K} / \mathrm{W}$. It clearly confirms that the Kapitza resistance between $\mathrm{Si}$ and solid ${ }^{4} \mathrm{He}$ is anomalous just as it is between $\mathrm{Si}$ and superfluid ${ }^{4} \mathrm{He}$. These findings also suggest that a more general phenomenon, other than the acoustic impedances, plays a key role in phonon transmission when two bodies are highly mismatched, irrespective of whether ${ }^{4} \mathrm{He}$ is in the superfluid or solid phase in our case.

In applying the AM model, the $\mathrm{Si} /$ solid ${ }^{4} \mathrm{He}$ interface is assumed to be atomically flat, with both solids being perfect crystals with their $c$ axes aligned perpendicularly to the interface. Under these conditions the scattering at the interface is purely of a specular nature. It is interesting to note that our experimental value can be retrieved if the critical cone angle 
in Eq. (4) is extended from $\theta_{c} \approx 3^{\circ}$ to $\theta_{c} \approx 17^{\circ}$. Increasing the critical angle implies "softening" the boundary condition on the continuity of the parallel components of the phonon wave vectors in the plane of the interface (see Supplemental Material). The transmission of phonons of longer wavelengths (and therefore smaller frequencies) is facilitated across the interface by a nonspecular (diffuse) scattering mechanism. This scenario is highly plausible since our Si crystal surface has roughnesses on the order of a few nanometers at scale lengths comparable to phonon wavelengths in solid ${ }^{4} \mathrm{He}$. We note that the lattice misfit at the interface also favors this scenario despite the fact that both the Si (111) and solid helium have hexagonal crystalline structures. In summary, not only does the nonspecular contribution constitute a parallel transmission channel, it predominates over specular scattering mechanisms just as for solid/superfluid ${ }^{4} \mathrm{He}$ interfaces.

\section{Diffuse mismatch (DM) model}

The DM model [15] describes a fully diffusive phonon scattering regime at the interface and the thermal boundary resistance is given by $R_{\mathrm{DM}}=$ $\frac{1}{2 a}\left[\left(1 / \sum_{j} c_{\mathrm{He}, j}^{-2}\right)+\left(1 / \sum_{j} c_{S i, j}^{-2}\right)\right] T^{-3}$. Using the velocities given above we estimate $R_{\mathrm{DM}} \approx 11.9 T^{-3} \mathrm{~cm}^{2} \mathrm{~K} / \mathrm{W}$ for the $\mathrm{Si} /$ solid ${ }^{4} \mathrm{He}$ interface. At $T=0.778 \mathrm{~K}, R_{\mathrm{DM}}=$ $25.3 \mathrm{~cm}^{2} \mathrm{~K} / \mathrm{W}$, which is $\sim 2$ times smaller than our measured value (see Fig. 3).

\section{Phonon radiation (PR) limit}

In the PR limit [16], phonons of all wavelengths in the less-dense material (medium 1) are elastically transmitted whereas the shorter-wavelength phonons in the dense material are reflected. Consequently, all thermal frequencies in medium 2 larger than the maximum frequency in medium 1 are cut off. Here, anharmonic/inelastic [17] phonon-scattering mechanisms are neglected. From Eq. (3), the thermal resistance in the phonon radiation limit for the $\mathrm{Si} /$ solid ${ }^{4} \mathrm{He}$ interface can therefore be written as $R_{\mathrm{rad}}=\left(4 a T^{3}\right)^{-1}\left[c_{\mathrm{He}, L}^{-2}+2 c_{\mathrm{He}, T}^{-2}\right]^{-1}=$ $0.0149 T^{-3} \mathrm{~cm}^{2} \mathrm{~K} / \mathrm{W}$. At $T=0.778 \mathrm{~K}$, the lower bound of the elastic thermal resistance is $R_{\mathrm{rad}}=0.0316 \mathrm{~cm}^{2} \mathrm{~K} / \mathrm{W}$. Works [18] claim that the PR model prediction tends to agree with experimental results for highly mismatched interfaces at low temperatures. Our experimental value exceeds both the DM and the PR model predictions. Finally, we note that anisotropy of the $R_{\mathrm{DM}}$ and $R_{\text {rad }}$ as a function of the ${ }^{4} \mathrm{He}$ crystal orientation with respect to the solid surface is negligible in our analysis (see Supplemental Material).

\section{Density of states and resonant scattering}

In our recent study [9] we demonstrated that the Adamenko and Fuks (AF) theory of resonant scattering of phonons makes a significant advancement in explaining the observed Kapitza resistance $R_{K, L}$ at the $\mathrm{Si}$ /superfluid interface. In the AF model the heat flux across an interface is amplified by factor $f \propto\left(\sigma / \lambda_{\mathrm{HeII}}\right)^{2}$. This arises from the surface resonant scattering mechanism which prevails when nanoscale surface roughness heights $\sigma$ preferentially interact with wavelengths from the incident phonon spectrum. Consequently, the thermal resistance decreases by a factor $R_{\mathrm{AM}} / R_{\sigma}=1+0.5 \gamma^{2} f$, where $R_{\sigma}$ is the thermal resistance calculated in the AF model for an isotropically rough surface with an average surfaceroughness inclination $\gamma=(2 \sigma / \ell)$, where $\ell$ is the roughness height-height correlation length. Since the sound velocity $c_{L}(P)$ in superfluid ${ }^{4} \mathrm{He}$ varies from $239 \mathrm{~m} / \mathrm{s}$ at saturated vapor pressure (SVP) to $362 \mathrm{~m} / \mathrm{s}$ at $P=24 \mathrm{bar}$, the dominant wavelength $\lambda_{\mathrm{HeII}}=\left(h c_{L}(P)\right) /\left(3.8 k_{B} T\right)$ correspondingly changes from $\lambda_{\mathrm{HeII}} \approx 3.86 \mathrm{~nm}$ at SVP to $\lambda_{\mathrm{HeII}} \approx 5.83 \mathrm{~nm}$ just before solidification at $T=0.778 \mathrm{~K}$. The presence of a Gaussian distribution of roughnesses assures that the resonant scattering criterion $\sigma=\lambda_{\mathrm{HeII}} / 3$ is fulfilled for all $\lambda_{\mathrm{HeII}}$ at all pressures, thereby explaining the rather small change in our $R_{K, L}$ measurements as seen in Fig. 3. We find that a $\sigma$ value of $\sim 1.9 \mathrm{~nm}$ suffices to explain the observed experimental value $R_{K, L}=(80 \pm 8) \mathrm{cm}^{2} \mathrm{~K} / \mathrm{W}$ at 24 bar.

Now in solid ${ }^{4} \mathrm{He}$, the dominant wavelengths of longitudinal and transverse thermal phonons vary as $\lambda_{\mathrm{He}, j}=$ $h c_{j}(\varphi) /\left(3.8 k_{B} T\right)$, where $\varphi$ is the ${ }^{4} \mathrm{He}$ crystal tilt angle with respect to the $c$ axis. Considering the variation of $c_{j}$ with $\varphi$ determined by Crepeau et al. [19], we estimate $\lambda_{\mathrm{He}, L} \leqslant 9.86$ $\mathrm{nm}$ and $\lambda_{\mathrm{He}, T} \leqslant 4.75 \mathrm{~nm}$ at $T=0.778 \mathrm{~K}$. These values are close to phonon wavelengths in superfluid ${ }^{4} \mathrm{He}$. We recall that $\lambda_{\text {HeII }}=5.83 \mathrm{~nm}$ at $0.778 \mathrm{~K}$. Atomic force microscopy (AFM) surface roughness analysis of our Si sample shows that surface roughnesses on the order of $\lambda_{\mathrm{He}, j} / 3$ are present. Consequently, the resonant scattering criterion $\sigma \approx \lambda_{\mathrm{He}, j} / 3$ is also fulfilled for the $\mathrm{Si} /$ solid ${ }^{4} \mathrm{He}$ case just as it is for $\mathrm{Si} /$ superfluid $\mathrm{He}$. We therefore conjecture that the origin of diffuse scattering can very well emerge from the resonant scattering mechanism of phonons discussed previously.

If resonant scattering is indeed the key thermal transfer mechanism at the interface, then the sharp drop in $R_{K}$ upon solidification must depend strongly on the difference in the number of phonon density of states accessible between solid and superfluid helium. We estimate the increase in the density of states due to the phase change of helium as follows. For solid ${ }^{4} \mathrm{He}$ the density of states in the frequency interval of $\omega$ is $g_{s}(\omega)=9 n_{s} \omega^{2} / \omega_{D}^{3}$, where $n_{s}$ is the number of atoms per unit volume and $\omega_{D}$ is the Debye frequency. Treating superfluid ${ }^{4} \mathrm{He}$ as a dilute Bose gas, the density of states can be written as $g_{L}(\omega)=3 n_{L} \omega^{2} / \omega_{D}^{3}$, where $n_{L}$ is the number density in the superfluid. The ratio $\left(g_{s} / g_{L}\right) \approx 3 \rho_{s} / \rho_{L}=3.3$, where $\rho_{L}=0.17 \mathrm{~g} / \mathrm{cm}^{3}$ is the superfluid density at $\sim 24 \mathrm{bar}$, just before solidification. The scattering mechanism being the same (resonant) before and after solidification, we expect $R_{K, S} \approx$ $R_{K, L} / 3 \approx 26 \mathrm{~cm}^{2} \mathrm{~K}^{4} / \mathrm{W}$ since there are two more modes now participating in phonon transmission. It is interesting to note that this value is in very good agreement with the DM model prediction calculated above.

\section{Influence of dislocations in solid ${ }^{4} \mathrm{He}$ \\ 1. Interaction phonon dislocations}

In order to make progress in the interpretation of our results, we present an analysis based on the presence of dislocations in solid ${ }^{4} \mathrm{He}$. The analysis brings to light a source of thermal resistance in bulk solid ${ }^{4} \mathrm{He}$ which corroborates the plausibility of the large discrepancy between our measured value and the $\mathrm{DM}$ and PR model predictions for the $\mathrm{Si} /$ solid ${ }^{4} \mathrm{He}$ interface. The analysis also provides a tentative explanation of the 
TABLE I. Expressions for thermal resistivity in $(\mathrm{mK} / \mathrm{W})$ determined for different scattering mechanisms. The numerical coefficients are calculated in SI units, with $N_{d}$ in $\mathrm{m}^{-2}$. The integral in Eq. (8) is calculated numerically with the upper limit determined using $x_{D}=\theta_{D} / T$, with $\theta_{D} \approx 25 \mathrm{~K}$.

\begin{tabular}{lll}
\hline \hline Mechanism & Relaxation time $\tau_{p}(\mathrm{~s})$ & Thermal resistivity $W_{p}(\mathrm{mK} / \mathrm{W})$ \\
\hline Core & $\tau_{c}^{-1}=V_{o}^{4 / 3} N_{d} \omega^{3} / v_{D}^{2}$ & $W_{c}=8.23 \times 10^{-15} N_{d} / v_{D}$ \\
Fluttering & $\tau_{F l}^{-1}=4.95 \times 10^{7} b^{2} v_{D}^{3} N_{d} / T^{3}$ & $W_{F l}=1.21 \times 10^{-3} v_{D}^{4} b^{2} N_{d} / T^{6}$ \\
Screw & $\tau_{s}^{-1}=0.06 N_{d} b^{2} \gamma^{2} \omega$ & $W_{s}=0.696 v_{D} b^{2} \gamma^{2} N_{d} / T^{2}$ \\
\hline \hline
\end{tabular}

thermal resistances measured at the $\mathrm{Cu} /$ solid ${ }^{4} \mathrm{He}$ interface, performed in two independent studies.

Dislocations are known to have an important effect on the thermal and mechanical properties of solids. Recent studies have shown that the large mobility of dislocations plays a decisive role in explaining an elastic anomaly called "giant plasticity" and thereby refute the supersolidity of ${ }^{4} \mathrm{He}$ crystals around $0.2 \mathrm{~K}$. From the findings of the group of Balibar [1-4] we infer that at our temperature of $T=0.778 \mathrm{~K},{ }^{3} \mathrm{He}$ impurities present in solid ${ }^{4} \mathrm{He}$ have no longer a pinning effect on dislocations and mobile dislocations flutter under collisions with thermal phonons. Following Granato and Lücke [20], Souris et al. [3] cast the scattering time for this fluttering process as $\tau_{F l}=B L^{2} / \pi^{2} C$, where $C \approx 1.7 \times 10^{7} b^{2}$ is the dislocation energy per unit length in $(\mathrm{J} / \mathrm{m})$ with $b$ the Burgers vector, $L$ the typical length between dislocations in the absence of a pinning effect due to ${ }^{3} \mathrm{He}$ impurities, and $B=14.4 k_{B}^{3} T^{3} / \pi^{2} \hbar^{2} v_{D}^{3}$ is the phonon damping coefficient derived by Ninomiya $[21,22]$ by considering the interaction of phonons at a given temperature with a mobile dislocation. In fact, the coefficient $B$ characterizes the momentum transfer from phonons to the mobile dislocation. The latter experiences a drag force per unit length $F_{D}=-B v_{\text {dis }}$, where the dislocation velocity $v_{\text {dis }}$ decreases as $T$ increases. Taking $L^{2} \approx 1 / N_{d}$, where $N_{d}$ is the dislocation density, the relaxation time simplifies to

$$
\tau_{F l}^{-1}=4.95 \times 10^{7} b^{2} v_{D}^{3} N_{d} / T^{3} .
$$

The numerical coefficient is due only to the physical constants and is given in SI units.

To highlight the importance of the fluttering mechanism, we recall that in ordinary solids the effective dislocation scattering rate $\tau_{d}^{-1}$ is generally the sum of two frequencydependent phonon-scattering processes occurring at (i) lattice distortion sites (called dislocation cores), characterized by $\tau_{c}^{-1}$ and (ii) outside the core within the elastic strain field (called screw dislocation), characterized by $\tau_{s}^{-1}$. Klemens [23] derived the scattering rates for both these cases. For a network of screw dislocations parallel to the plane of the interface and perpendicular to the temperature gradient, he showed $\frac{1}{\tau_{s}}=\sqrt{2^{3} / 3^{7}} N_{d} b^{2} \gamma^{2} \omega\left[\frac{1}{2}+\frac{1}{24}\left(\frac{1-2 v}{1-v}\right)^{2}\left\{1+\sqrt{2}\left(\frac{c_{L}}{c_{T}}\right)^{2}\right\}^{2}\right]$, where $\gamma$ and $v$ are, respectively, the Grüneisen constant and the Poisson ratio. Taking $v=1 / 3$ and noting that $\left(c_{\mathrm{He}, T} / c_{\mathrm{He}, L}\right) \approx$ 0.5 , the above equation simplifies to

$$
\tau_{s}^{-1}=0.06 b^{2} \gamma^{2} \omega N_{d}
$$

The scattering rate from the dislocation core is expressed as

$$
\tau_{c}^{-1}=V_{o}^{4 / 3} \omega^{3} N_{d} / v_{D}^{2},
$$

where the hcp unit-cell volume $V_{o}=2^{1 / 2} 3 a^{3}$, with $a=0.367$ $\mathrm{nm}$ being the basal lattice constant of solid ${ }^{4} \mathrm{He}$.

The lattice thermal resistivity $W_{p}$ due to type- $p$ dislocations (where $p$ refers to core, screw, and fluttering dislocations) in an isotropic dielectric solid is given, in the Debye approximation, by $W_{p}^{-1}=\frac{1}{3} \sum_{j} \int \hbar \omega_{j} \frac{\partial N\left(\omega_{j}, T\right)}{\partial T} c_{j}^{2} \tau_{p} d \omega_{j}$, where $\tau_{p}^{-1}$ is the scattering rate. Now, letting $x=2 \pi h \omega / k_{B} T$ and introducing the Debye velocity $v_{D}$ given by $3 v_{D}^{-3}=\sum_{j} c_{j}^{-3}$, the inverse thermal resistivity becomes

$$
W_{p}^{-1}=\frac{4 \pi k_{B}}{v_{D}}\left(\frac{k_{B}}{h}\right)^{3} T^{3} \int_{0}^{x_{D}} \tau_{p}(x) \frac{x^{4} e^{x}}{\left(e^{x}-1\right)^{2}} d x .
$$

In the determination of $v_{D}$, the velocities $c_{j}$ of each branch are averaged over all angles. Table I shows the expressions of $W_{p}$ we found for each scattering mechanism. In Fig. 4 we plotted the thermal resistivity normalized with respect to the dislocation density, as a function of temperature for each scattering mechanism. For solid ${ }^{4} \mathrm{He}$, we took the Burgers vector $b=0.367 \mathrm{~nm}$ (Ref. [2]), $\gamma=2.7$ (Ref. [24]), and $v_{D}=285 \mathrm{~m} / \mathrm{s}$ (Ref [19]). This figure clearly illustrates that $\left(W_{F l} / N_{d}\right)$ is almost four orders of magnitude stronger com-

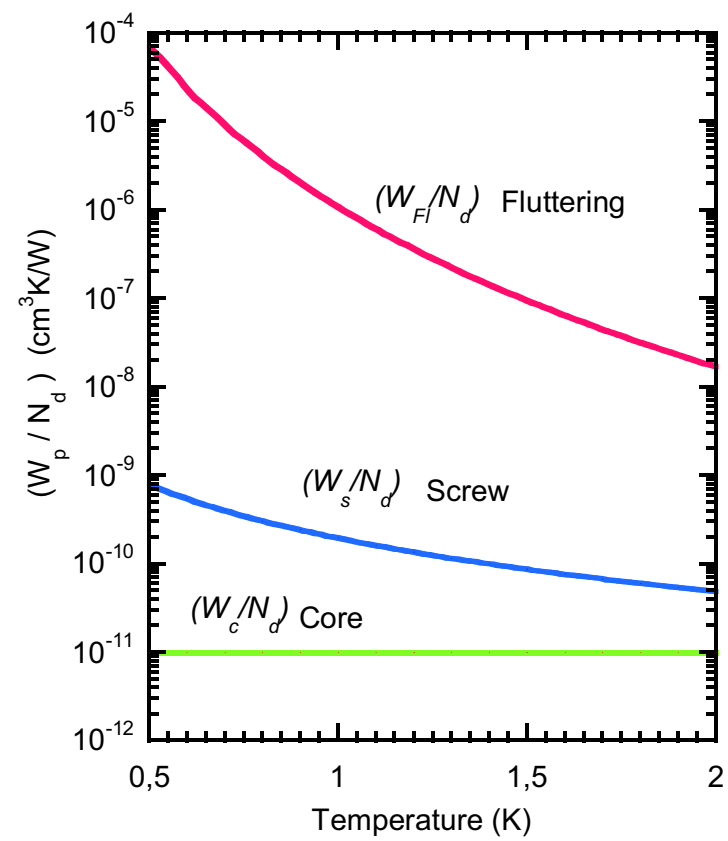

FIG. 4. Thermal resistivities normalized with respect to the dislocation density (see Table I) for the interaction of phonons with dislocations. The dominant contribution is due to the fluttering mechanism described in Refs. [21,22]. 


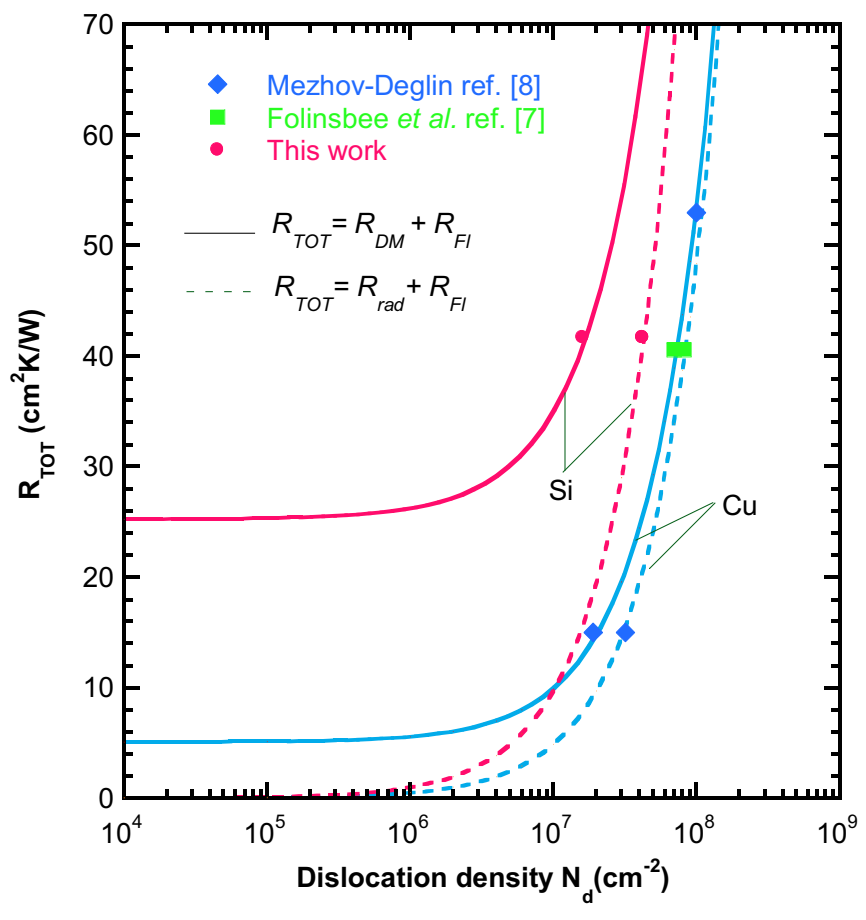

FIG. 5. The solid and dashed curves represent, respectively, $R_{\mathrm{DM}}+R_{F l}$ and $R_{\mathrm{rad}}+R_{F l}$ at $T=0.778 \mathrm{~K}$. Pink curves are for $\mathrm{Si} /$ solid $^{4} \mathrm{He}$ and blue curves are for $\mathrm{Cu} /$ solid $^{4} \mathrm{He}$. The data points are placed on the curves to show the dislocation densities which explain $R_{K S}$ measurements.

pared to $\left(W_{c} / N_{d}\right)$ and $\left(W_{s} / N_{d}\right)$ at $T<1 \mathrm{~K}$. Consequently, the influence of core and screw dislocations can therefore be neglected for simplicity hereon.

Unlike scattering from core and screw dislocations, the fluttering mechanism is frequency independent. Therefore phonons of all wavelengths participate in transferring, even partially, their momentum to dislocations.

In our experimental configuration the thermometer measuring the temperature in solid ${ }^{4} \mathrm{He}$ is situated at a distance of $\ell_{\mathrm{He}} \approx 0.2 \mathrm{~cm}$ from the interface. The thermal resistance due to the fluttering mechanism is therefore given by $R_{F l}=$ $W_{F l} \ell_{\mathrm{He}}=2.13 \times 10^{-7} N_{d} / T^{6} \mathrm{~cm}^{2} \mathrm{~K} / \mathrm{W}$, where $N_{d}$ is now in $\mathrm{cm}^{-2}$.

To demonstrate the impact of dislocations on our measurement, we plot in Fig. 5 the quantities $R_{\mathrm{DM}}+R_{F l}$ and $R_{\text {rad }}+R_{F l}$ as a function of $N_{d}\left(\right.$ in $\left.^{-2}\right)$ at $T=0.778 \mathrm{~K}$. The solid and dashed curves in pink depict the excess thermal resistance for the $\mathrm{Si} / \mathrm{solid}{ }^{4} \mathrm{He}$ interface with the limiting values being, respectively, $R_{\mathrm{DM}}=25.3 \mathrm{~cm}^{2} \mathrm{~K} / \mathrm{W}$ and $R_{\mathrm{rad}}=$ $0.0316 \mathrm{~cm}^{2} \mathrm{~K} / \mathrm{W}$. The key result here is that our experimental data (pink dots) are retrieved for a dislocation density $N_{d}=$ $1.5 \times 10^{7} \mathrm{~cm}^{-2}$ when the limit is given by the DM model. This dislocation density increases by a factor of $\sim 3$ only when the phonon radiation value is considered to be the alternate limit. For $N_{d}<10^{6} \mathrm{~cm}^{-2}$ the thermal resistance contribution due to dislocations is negligible. On the contrary, for $N_{d}>10^{8} \mathrm{~cm}^{-2}$ the thermal impedance due to the fluttering mechanism predominates over the limiting values of the two models. This clearly indicates that the measurements are highly dependent on the solid ${ }^{4} \mathrm{He}$ crystal quality.

\section{Comparison to the $\mathrm{Cu}$-solid ${ }^{4} \mathrm{He}$ interface}

To our knowledge previous studies conducted were for copper/solid ${ }^{4} \mathrm{He}$ interfaces by Mezhov-Deglin [8] and Folinsbee and Anderson [7]. We examine their experimental values at the minimum temperature $T=0.778 \mathrm{~K}$. Folinsbee and Anderson found $R_{K}^{F} \approx 40.6 \mathrm{~cm}^{2} \mathrm{~K} / \mathrm{W}$ whereas Mezhov-Deglin measured two distinct values, namely $R_{K}^{M 1} \approx 53 \mathrm{~cm}^{2} \mathrm{~K} / \mathrm{W}$ for a chemically polished and annealed Cu sample; and $R_{K}^{M 2} \approx$ $15 \mathrm{~cm}^{2} \mathrm{~K} / \mathrm{W}$ for very pure $\mathrm{Cu}$ sample annealed in high vacuum. Since these $\mathrm{Cu}$ surfaces are not atomic planes, they must have roughnesses less than $\sim 3.5 \mathrm{~nm}$ on scale lengths of $\lambda_{\mathrm{He}, j}$ which satisfy the resonant scattering condition $\sigma=\lambda_{\mathrm{He}, j} / 3$ discussed above. Further, the striking common feature in all these experiments is that the measured values of the thermal boundary resistances remain the same (within experimental error), independent of whether helium is in the superfluid or the solid phase. These puzzling results hint that a thermal contribution from some other mechanism emerges after solidification of superfluid ${ }^{4} \mathrm{He}$ and compensates the drop in the thermal boundary resistance.

In Fig. 5 the blue solid and dashed curves show the evolution of the effective thermal resistance for $\mathrm{Cu} / \mathrm{solid}^{4} \mathrm{He}$ interface as a function of $N_{d}$. The two limiting values are given by $R_{\mathrm{DM}}=$ $5.1 \mathrm{~cm}^{2} \mathrm{~K} / \mathrm{W}$ and $R_{\text {rad }}=0.0316 \mathrm{~cm}^{2} \mathrm{~K} / \mathrm{W}$ at $T=0.778 \mathrm{~K}$. For these resistances we took $\ell_{\mathrm{He}} \approx 0.1 \mathrm{~cm}$, corresponding to the thickness of the solid layer in these experiments. As seen from Fig. 5, dislocation densities in the range $10^{7}$ to $10^{8} \mathrm{~cm}^{-2}$ once again suffice to fully compensate the decrease in the thermal boundary resistance with solidification as can be seen by their data points on these curves. In summary, there is a critical dislocation density above which $R_{F l}$ has a detectable influence on the measurements of $R_{K S}$.

\section{Dislocation densities}

We note that the dislocation densities that we determined may be overestimated by an order of magnitude. This is so because we set $N_{d} \approx 1 / L^{2}$ in the expression leading to Eq. (5) for the relaxation time $\tau_{F l}$. In fact, the product $N_{d} L^{2}$ represents a geometrical parameter which characterizes the dislocation network connectedness. Since $L$ depends on $N_{d}$, $N_{d} L^{2}$ increases with improvement in the quality of the ${ }^{4} \mathrm{He}$ crystal to reach values of $\sim 20-60$ [25]. Our crystals were grown at the minimum of the melting curve and remained in equilibrium with the superfluid phase. These conditions strongly suggest that our solid ${ }^{4} \mathrm{He}$ is a high-quality single crystal and therefore $N_{d} L^{2}$ can be greater than 1. Consequently, our $N_{d}$ values represent an upper limit.

One may hypothesize that the quality of the interface in our case may be imperfect owing to pockets of superfluid. In such a case, it is clear that the intrinsic value of the thermal interface resistance between $\mathrm{Si}$ and solid ${ }^{4} \mathrm{He}$ must be smaller than $R_{\mathrm{DM}}$ and greater than $R_{\mathrm{rad}}$, the latter being the lower limit. This is why we considered both these thermal resistances in determining the dislocation densities. The difference in the dislocation densities obtained using these limits vary at most by a factor of approximately three as found above and therefore do not alter our conclusions.

The dislocation densities on the order of $10^{7} \mathrm{~cm}^{-2}$ which we have extracted from our measurements (see Fig. 5) are 
very consistent with those found in good-quality crystals grown at constant pressure and temperature as in our case. This is in support of the robustness of our analysis as $N_{d}$ does not appear to depend on the concentration of ${ }^{3} \mathrm{He}$ impurities above $0.6 \mathrm{~K}[26]$.

\section{CONCLUSION}

We experimentally show that a first-order transition occurs in the Kapitza resistance at the superfluid ${ }^{4} \mathrm{He} / \mathrm{Si}$ (111) interface upon crystallization of ${ }^{4} \mathrm{He}$ at the minimum of the melting curve. Using our modified acoustic mismatch model we demonstrate a predominant contribution from diffuse scattering outside the critical angle in the less-dense medium. The bulk acoustic impedances of each medium do not control phonon transmission, in complete discord with the AM theory [27] for solid/solid interfaces. As established for solid/superfluid interfaces, we show that the same roughnessphonon wavelength criterion for the surface resonant scattering is fulfilled for solid/solid ${ }^{4} \mathrm{He}$ interfaces. We argue that the drop in the measured values of the Kapitza resistance at the $\mathrm{Si} /$ solid ${ }^{4} \mathrm{He}$ interface is less than expected considering the higher density of states available in solid ${ }^{4} \mathrm{He}$ compared to the superfluid. We then explain our measurements by demonstrating that the interaction of thermal phonons with vibrating dislocations within solid ${ }^{4} \mathrm{He}$ dramatically influences the thermal interface resistance. We clearly identify the phonon fluttering mechanism and exclude the influence of screw and cores dislocations. We show that densities greater than $\sim 10^{7} \mathrm{~cm}^{-2}$ suffice to overshadow the interface thermal resistance. Dislocation densities less than $\sim 10^{6} \mathrm{~cm}^{-2}$ have no effect on the intrinsic values on the thermal interface resistance. The present analysis provides a consistent interpretation of the measurements of the thermal resistance between $\mathrm{Cu}$ and solid ${ }^{4} \mathrm{He}$, conducted independently by Folinsbee and Anderson, and Mezhov-Deglin.
Further experiments in a wide temperature range are necessary to establish the temperature dependency of the Kapitza resistance between solid ${ }^{4} \mathrm{He}$ (a quantum solid) and $\mathrm{Si}$ (a classical solid). In particular it would be interesting to examine the evolution in the thermal resistance at $T<0.3 \mathrm{~K}$ where important changes in shear modulus occur, leading to the giant plasticity of solid ${ }^{4} \mathrm{He}$. At these low temperatures the fluttering mechanism disappears and dislocations move without dissipation. At slightly lower temperatures (below $0.1 \mathrm{~K}$ ) the dislocations are immobilized by ${ }^{3} \mathrm{He}$ impurity binding. Consequently, we would expect changes in the thermal interface resistance $R_{K S}$ which shall serve to corroborate our interpretation.

Finally, the impact of dislocations at interfaces between classical materials at nanoscale and in nanowire structures are still at an infancy stage. The study of the interface between a classical solid and solid ${ }^{4} \mathrm{He}$ helps unveil the role of dislocations [28] which impacts the tailoring of thermal interface resistance as we have demonstrated.

\section{ACKNOWLEDGMENTS}

The experiments were conducted at the Institut de Physique Nucléaire d'Orsay (IPNO-CNRS). We acknowledge technical assistance of F. Dubois. The authors express their thanks to Dr. J. Bossy for comments on crystal growth, Prof. L. P. Mezhov-Deglin for a fruitful exchange on his experiments, Prof. H. J. Maris for discussions and suggestions on future experiments, and Prof. B. Castaing for a comment which inspired this work. This work has benefited from the financial support of the LabeX LaSIPS (ANR-10-LABX-0040-LaSIPS) managed by the French National Research Agency under the "Investissements d'avenir" program (Grant No. ANR-11-IDEX-0003-02).
[1] A. Haziot, X. Rojas, A. D. Fefferman, J. R. Beamish, and S. Balibar, Phys. Rev. Lett. 110, 035301 (2013).

[2] A. Haziot, A. D. Fefferman, F. Souris, J. R. Beamish, H. J. Maris, and S. Balibar, Phys. Rev. B 88, 014106 (2013).

[3] F. Souris, A. D. Fefferman, H. J. Maris, V. Dauvois, P. Jean-Baptiste, J. R. Beamish, and S. Balibar, Phys. Rev. B 90, 180103(R) (2014).

[4] S. Balibar, J. Beamish, A. Fefferman, A. Haziot, X. Rojas, and F. Souris, C. R. Phys. 17, 264 (2016).

[5] A. D. Fefferman, F. Souris, A. Haziot, J. R. Beamish, and S. Balibar, Phys. Rev. B 89, 014105 (2014).

[6] M. L. Dos Reis, A. Choudhury, and L. Proville, Phys. Rev. B 95, 094103 (2017).

[7] J. Folinsbee and A. Anderson, Phys. Rev. Lett. 31, 1580 (1973).

[8] L. P. Mezhov-Deglin, Sov. Phys. JETP 44, 761 (1976).

[9] A. Ramiere, S. Volz, and J. Amrit, Nat. Mater. 15, 512 (2016).

[10] I. N. Adamenko and I. M. Fuks, Sov. Phys. JETP 32, 1123 (1971).

[11] J. Amrit, Phys. Rev. B 81, 054303 (2010).
[12] E. R. Grilly, J. Low Temp. Phys. 11, 33 (1973).

[13] J. Bossy (private communication).

[14] See Supplemental Material at http://link.aps.org/supplemental/ 10.1103/PhysRevB.97.014308 for a simplified acoustic mismatch model for strongly mismatched solids, and the dependence of the thermal interface resistance on the ${ }^{4} \mathrm{He}$ crystal orientation. The Supplemental Material includes Ref. [19].

[15] E. T. Swartz and R. O. Pohl, Rev. Mod. Phys. 61, 605 (1989).

[16] R. J. Stoner and H. J. Maris, Phys. Rev. B 48, 16373 (1993).

[17] K. Sääskilahti, J. Oksanen, J. Tulkki, and S. Volz, Phys. Rev. B 90, 134312 (2014).

[18] G. T. Hohensee, R. B. Wilson, and D. G. Cahill, Nat. Commun. 6, 6578 (2015).

[19] R. H. Crepeau, O. Heybey, D. M. Lee, and S. A. Strauss, Phys. Rev. A 3, 1162 (1971).

[20] A. Granato and K. Lücke, J. Appl. Phys. 27, 583 (1956).

[21] T. Ninomiya, J. Phys. Soc. Jpn. 36, 399 (1974). 
[22] T. Ninomiya, Scr. Metall. 18, 669 (1984).

[23] P. G. Klemens, Proc. Phys. Soc. Sect. A 68, 1113 (1955).

[24] D. S. Greywall, Phys. Rev. B 16, 5127 (1977).

[25] E. Varoquaux, Phys. Rev. B 86, 064524 (2012).
[26] F. Souris, A. D. Fefferman, A. Haziot, N. Garroum, J. R. Beamish, and S. Balibar, J. Low Temp. Phys. 178, 149 (2015).

[27] I. M. Khalatnikov, An Introduction to the Theory of Superfludity (Addison-Wesley, New York, 1988).

[28] B. Castaing, Physics 6, 5 (2013). 